\section{Síndrome de DRESS inducido por sulfasalazina. ¿Consideramos esta reacción adversa grave al indicar el fármaco? Caso clínico}

\author{
EDITH PÉREZ DE ARCE ${ }^{1, a}$, RODRIGO QUERA², \\ LILIAN FLORES ${ }^{2, b}$, RODRIGO BLAMEY ${ }^{3}$
}

\section{DRESS syndrome induced by sulfasalazine. Report of one case}

\begin{abstract}
DRESS syndrome (Drug Reaction with Eosinophilia and Systemic Symptoms) is a severe, rare and potentially lethal idiosyncratic condition associated with the use of some drugs. Given its broad spectrum of presentation, clinical suspicion is essential for management, since it requires the immediate withdrawal of the culprit drug, support measures and the use of corticosteroids as the first line of treatment. We report a 24-year-old woman with a diagnosis of ulcerative colitis with joint involvement despite the use of infliximab, who presented symptoms, signs and laboratory compatible with DRESS syndrome on the third week after indicating sulfasalazine for her baseline disease.
\end{abstract}

(Rev Med Chile 2020; 148: 1213-1218)

Key words: Colitis, Ulcerative; Drug Hypersensitivity Syndrome; Sulfasalazine.

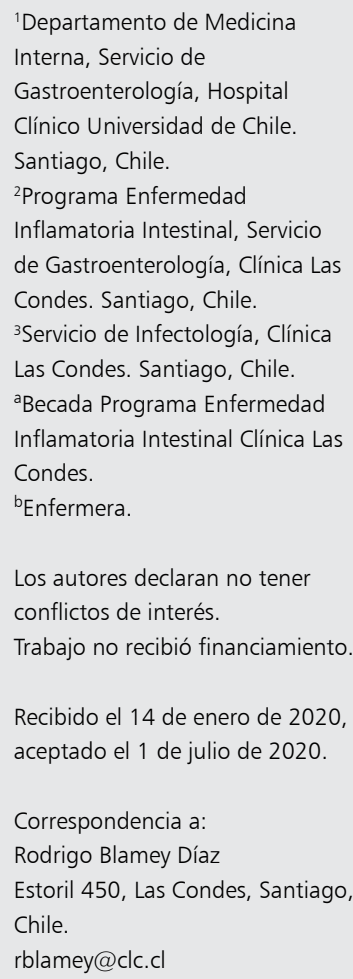

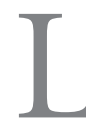

a reacción a drogas con eosinofilia y síntomas sistémicos o síndrome de DRESS (del inglés Drug Reaction with Eosinophilia and Systemic Symptoms) es una reacción grave e idiosincrática a drogas del tipo hipersensibilidad retardada, muy poco frecuente y potencialmente letal ${ }^{1,2}$. Aunque su presentación clínica es variada ${ }^{3}$, clásicamente se presenta con exantema o eritema cutáneo, fiebre, linfoadenopatías, eosinofilia y compromiso visceral (hepático, pulmonar, renal o cardíaco, principalmente) que ocurre en el transcurso de 2 a 6 semanas desde la introducción del fármaco, sin embargo, puede presentarse más precozmente con la reintroducción del mismo ${ }^{1,4}$. Actualmente existe una extensa lista de fármacos asociados al síndrome de DRESS (Tabla 1). A continuación, discutimos un caso clínico en relación al uso de sulfasalazina.

\section{Caso clínico}

Mujer de 24 años con antecedentes de colitis ulcerosa izquierda diagnosticada en 2016. Desde su diagnóstico mantuvo actividad inflamatoria pese al uso de mesalazina oral 4,5 g/día y local (enemas y supositorios) y azatioprina $175 \mathrm{mg} /$ día. Por inmunorefractariedad a tiopurínicos inició infliximab en dosis estándar, logrando remisión profunda. En agosto de 2019, luego de tres años de tratamiento, se suspendió azatioprina, manteniendo remisión clínica, con calprotectina fecal de $71 \mu \mathrm{g} / \mathrm{g}$. Evolucionó con artralgias de rodillas y tobillos que aparecieron 4 a 6 semanas después de la dosis de infliximab. Por persistencia de síntomas articulares, se inició sulfasalazina $2 \mathrm{~g} /$ día. A las 3 semanas, la paciente notó aumento de volumen cervical compatible con adenopatías $>20$ 
$\mathrm{mm}$ (Figura 1), fiebre intermitente hasta $38,5^{\circ} \mathrm{C}$, exantema en tronco, cuello y tobillos, y prurito plantar. Exámenes de laboratorio evidenciaban alteración del perfil hepático (bilirrubina total/ directa 0,16/0,12 mg/dL; GOT/GPT 222/386 U/L;

\section{Tabla 1. Fármacos frecuentemente asociados a} síndrome de DRESS

Anticonvulsivantes
- Carbamazepina, fenobarbital, fenitoína, ácido valproico,
lamotrigina
Alopurinol
Sulfonamidas
- Sulfasalazina, sulfadiazina, dapsona, trimetoprim-
sulfametoxazol
Antimicrobianos
- Amoxicilina, vancomicina, minociclina, trimetoprim-
sulfametoxazol, ampicilina/sulbactam

FA/GGT 105/68 U/L) y hemograma con leucocitos de $6.600 / \mathrm{mm}^{3}$ con $14,1 \%$ de eosinófilos $(924$ eosinófilos $/ \mathrm{mm}^{3}$ ). La serología para virus Epstein Barr, citomegalovirus, virus herpes, toxoplasma, bartonella, ELISA para VIH y RPR para sífilis fueron negativos. Por sospecha de linfoma en contexto de inmunosupresión crónica fue evaluada por hematología, descartando un cuadro linfoproliferativo. Reevaluando su historia clínica y farmacológica reciente, se decidió suspender sulfasalazina a las 6 semanas de su inicio, evolucionando con desaparición inmediata del eritema cutáneo y fiebre y disminución progresiva de las adenopatías cervicales, que desaparecieron al $6^{\circ}$ día de la suspensión. Debido a la persistencia de los síntomas articulares y bajos niveles plasmáticos de infliximab $(1,86 \mu \mathrm{g} / \mathrm{mL})$ con ausencia de anticuerpos, se optimizó la dosis a $5 \mathrm{mg} / \mathrm{kg}$ cada 6 semanas y se reinició azatioprina. Los exámenes de laboratorio mostraron ausencia de eosinófilos y normalización del perfil hepático.
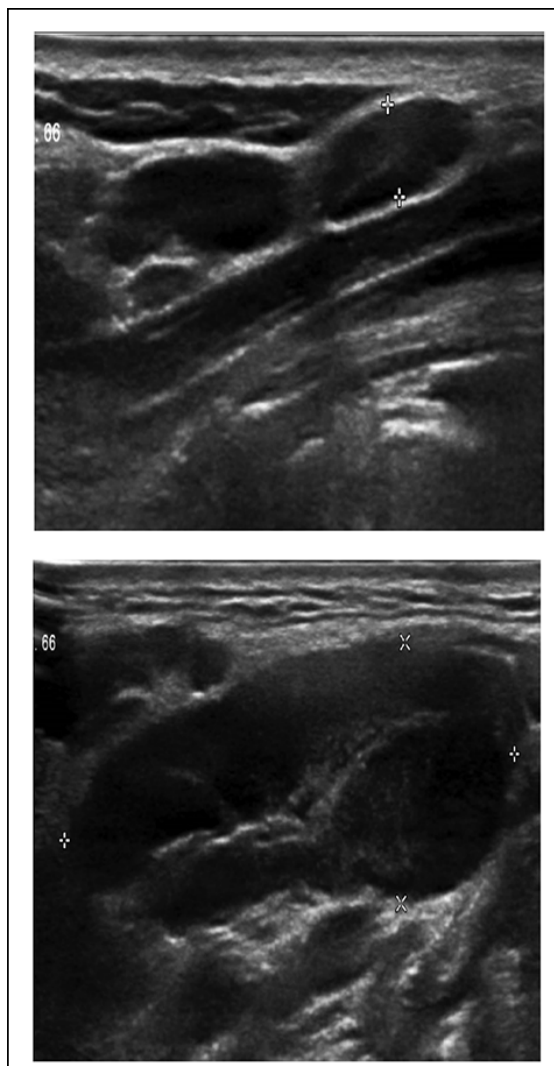
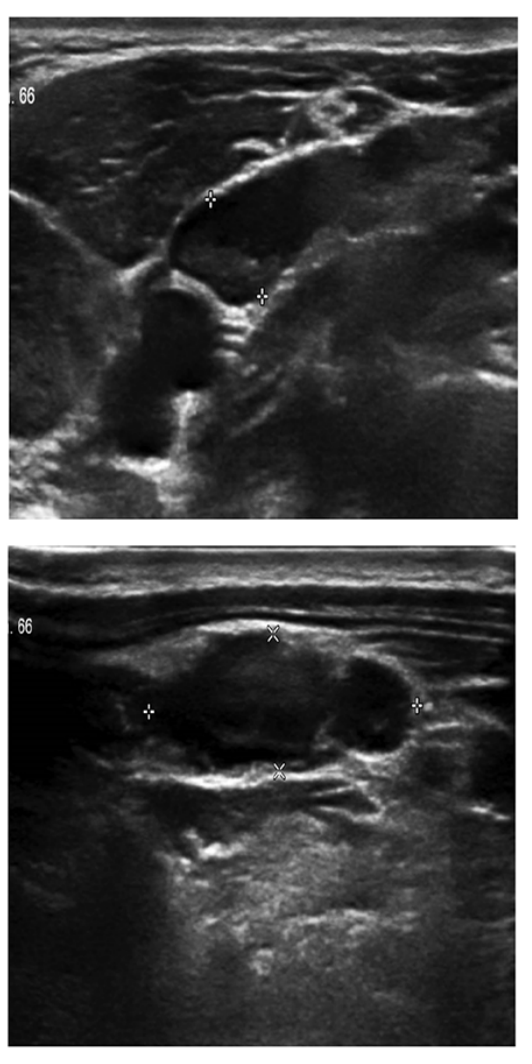

Figura 1. Ecografía partes blandas a las 3 semanas de inicio de sulfasalazina: múltiples adenopatías cervicales localizadas en grupos II de hasta $32 \times 16 \mathrm{~mm}$ a izquierda y 43 $x 15 \mathrm{~mm}$ a derecha, así como también en grupos III y $\mathrm{V}$ de hasta $24 \times 8 \mathrm{~mm}$ a derecha. 


\section{Discusión}

La primera descripción del término "síndrome de DRESS" fue realizada por Bocquet en 1996, quien simplificó la nomenclatura de los síndromes por hipersensibilidad a drogas y unificó criterios clínicos ${ }^{1}$. Aunque se desconoce su incidencia real, se estima una frecuencia de 1 a 10 en 10.000 exposiciones a drogas ${ }^{5,6}$, y en Estados Unidos de Norteamérica su prevalencia alcanza a 2,18 casos por 100.000 pacientes $^{7}$. Se presenta más frecuentemente en adultos, con una relación hombre:mujer de $0,8^{8}$. La mortalidad es cercana a $10 \%$, principalmente por la aparición de falla hepática fulminante ${ }^{1}$.

Aunque su etiopatogenia aún es desconocida, se han involucrado factores de predisposición genética, reactivación de infecciones virales ${ }^{9,10}$ (principalmente virus herpes humano $[\mathrm{VHH}]$ 6 y 7, Epstein Barr y citomegalovirus [CMV]) y alteraciones en el metabolismo de fármacos, como acetilación lenta y defectos en la detoxificación que resultan en metabolitos reactivos capaces de desencadenar una respuesta inmune alterada ${ }^{2}$. Entre los fármacos clásicamente asociados a este síndrome se describen anticonvulsivantes (35\%), alopurinol (18\%), sulfas (12\%) y antibióticos $(11 \%)^{8}$ (Tabla 1$)$, aunque en estudios más recientes, los antibióticos ocupan el primer lugar $(74 \%)^{7}$ $\mathrm{y}$, en una serie de pacientes reumatológicos, la sulfasalazina ocupó el segundo lugar luego del alopurinol $^{11}$.

Linfocitos activados (T CD4+ y T citotóxicas) y eosinófilos son los principales implicados en la inflamación sistémica y cutánea en DRESS y se han identificado en biopsias de piel y órganos afectados $^{12}$. El cuadro clásico corresponde a un exantema maculopapular difuso que compromete $>50 \%$ de la superficie corporal y que persiste a pesar del retiro del fármaco. Edema facial o periorbitario, púrpura, pústulas o eritrodermia también han sido descritos ${ }^{3,4,13}$, sin embargo, las manifestaciones cutáneas son heterogéneas y en algunos casos poco evidentes, reportándose entre $84 \%$ y $100 \%$ según algunas series ${ }^{3,8,13}$. Los síntomas sistémicos suelen presentarse hasta dos semanas antes del exantema con fiebre $>38^{\circ} \mathrm{C}$, síntomas flu-like, linfoadenopatías cervicales, axilares o inguinales y prurito (50\% presente al diagnóstico). Se ha descrito un empeoramiento paradojal de los síntomas tras el retiro del fármaco responsable ${ }^{4}$, situación que no ocurrió en nuestra paciente.

Dentro de las alteraciones hematológicas, el hallazgo más frecuente es la eosinofilia hasta $95 \%$ de los casos, leucocitosis y linfocitos atípicos al frotis $(30 \%)^{8}$. Hallazgos menos frecuentes son la trombocitopenia, anemia, neutropenia y el síndrome hemofagocítico.

El compromiso visceral determina la morbilidad y mortalidad del cuadro, siendo el hepático el más frecuente $(80 \% \text { de los casos })^{8}$, pudiendo reflejarse principalmente por una elevación de la alanino aminotransferasa o GPT (80\% de los casos) o como un cuadro colestásico, hepatomegalia, hepatitis anictérica o evolucionar hasta una falla hepática fulminante. Otras manifestaciones del compromiso visceral son la carditis, nefritis, neumonitis intersticial, encefalitis, meningitis, colitis, tiroiditis y compromiso pancreático, entre otros ${ }^{8}$. Nuestra paciente solo presentó alteración del perfil hepático de tipo hepatítico, que se resolvió tras el retiro de sulfasalazina.

La sulfasalazina, fármaco de la familia de las sulfonamidas, es ampliamente utilizada en reumatología para enfermedades articulares, y en gastroenterología como terapia en la enfermedad inflamatoria intestinal, especialmente con compromiso articular. El síndrome de DRESS inducido por sulfasalazina es conocido como el "síndrome de sulfasalazina de la semana tres"13, semana donde se inician los primeros síntomas (fiebre, linfoadenopatías, exantema y hepatomegalia), como aconteció en nuestro caso. Diferencias individuales en el metabolismo de las sulfonamidas pueden predisponer a reacciones idiosincráticas tipo DRESS: el fenotipo acetilador lento y una forma del citocromo P450 estarían involucrados en la generación de metabolitos potencialmente tóxicos (hidroxilamina) de las sulfonamidas, que aumentarían la susceptibilidad de los linfocitos in vitro, y el riesgo de hipersensibilidad a drogas ${ }^{14,15}$.

Debido al espectro de presentación del síndrome de DRESS, se debe tener un alto índice de sospecha, ya que existe una serie de diagnósticos diferenciales a considerar, como linfomas, enfermedades del tejido conectivo (lupus eritematoso sistémico, enfermedad de Kawasaki), infecciones virales (mononucleosis infecciosa, VIH, etc.), linfoadenopatía inmunoblástica y síndrome hipereosinofílico ${ }^{13}$. El antecedente de tratamiento con infliximab como inmunosupresor en nuestra 
paciente, nos llevó a descartar inicialmente un cuadro infeccioso o un síndrome linfoproliferativo.

Naranjo y cols. propusieron un algoritmo general que estima la causalidad de una reacción adversa a fármacos, y que recomendamos como una aproximación inicial al síndrome ${ }^{16}$. Sin embargo, dado que no existe un gold standard para el diagnóstico de síndrome de DRESS, el Registro
Europeo de Reacciones Adversas Cutáneas Graves (RegiSCAR $)^{8}$ y el Comité Japonés de Investigación en Reacción Adversa Cutánea Grave (J-SCAR) ${ }^{9}$ han propuesto criterios diagnósticos (Tabla 2) utilizados habitualmente para la comunicación de casos, pero que no están diseñados para excluir otras enfermedades con manifestaciones similares. Según RegiSCAR, la paciente cumplió criterios

Tabla 2. Criterios diagnósticos para síndrome de DRESS del Registro Europeo de Reacciones Adversas Cutáneas Graves (RegiSCAR) y el Comité Japonés de Investigación en Reacción Adversa Cutánea Grave (J-SCAR)

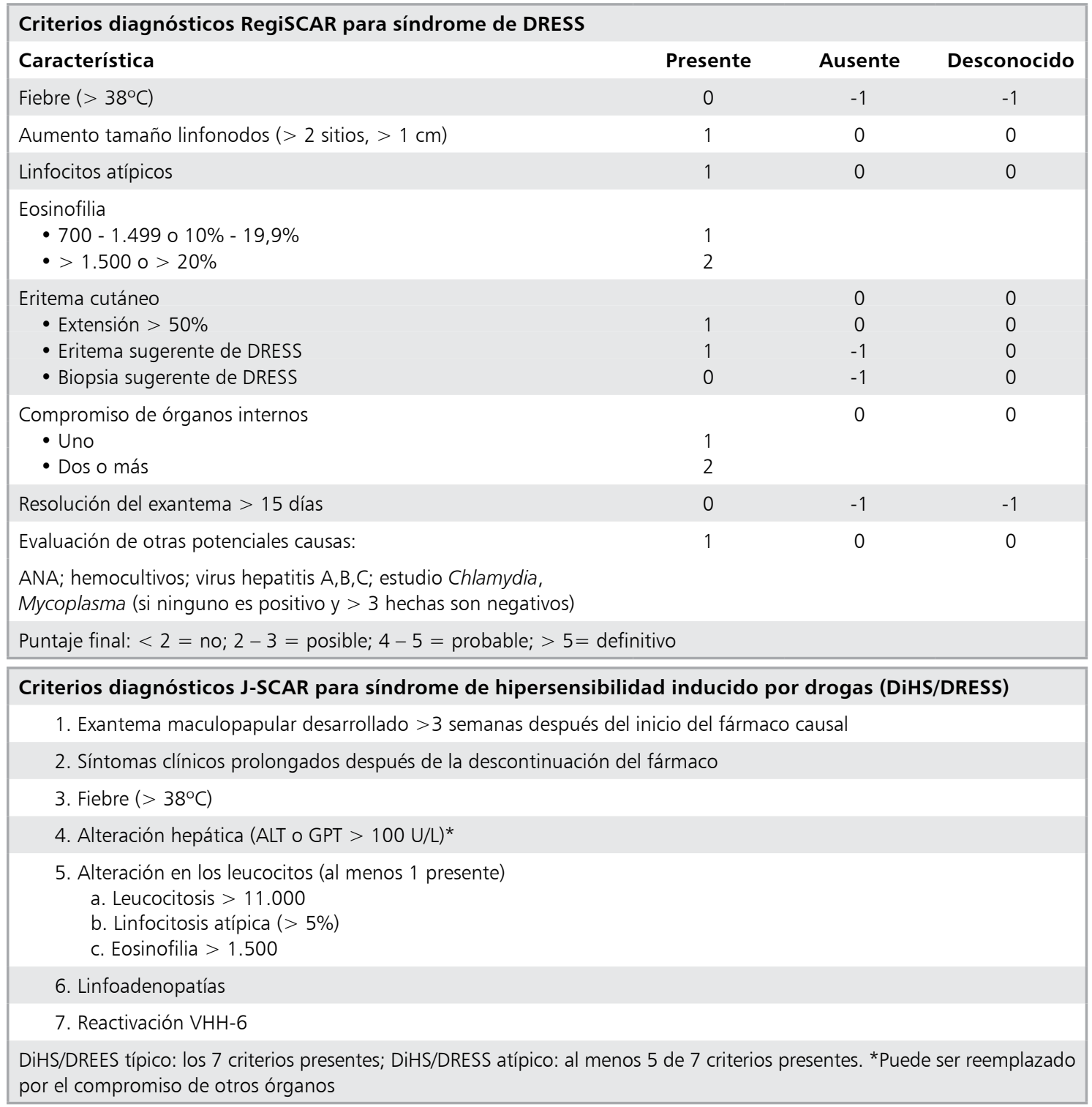


de "caso probable" y para J-SCAR, criterios de "DRESS atípico". Recientemente Mizukawa y cols. desarrollaron un sistema de puntuación para evaluar la gravedad y predecir el pronóstico de pacientes con síndrome de DRESS ${ }^{17}$.

El estudio de ciertos alelos del antígeno leucocitario humano (HLA-I) se han reportado como un factor de riesgo genético para las reacciones cutáneas severas a drogas específicas como es el caso de DRESS por alopurinol, lamotrigina y carbamazepina $^{12}$, sin embargo, su influencia en el cuadro clínico aún se investiga. Un avance en los métodos diagnósticos es el ensayo de inmunospot ligado a enzimas (ELISpot) que detecta la liberación de interferón (IFN) $-\gamma$ desde linfocitos estimulados específicamente con determinadas drogas, cuyo uso ha sido reportado en casos de DRESS por sulfasalazina ${ }^{18,19}$.

En relación al tratamiento, se requiere suspensión inmediata del fármaco sospechoso. El uso de corticoides orales (prednisona $1 \mathrm{a} 2 \mathrm{mg}$ / $\mathrm{kg}$, día o equivalente) o metilprednisolona en los casos más graves $(500 \mathrm{mg} /$ día ev por 3 días) con descenso gradual de dosis, ha sido recomendado con resultados controversiales ${ }^{20}$. Como tratamientos de segunda línea, existen reportes de $\mathrm{N}$-acetilcisteína ${ }^{21}$, inmunoglobulina $\mathrm{ev}^{22}$, ciclosporina $^{23}$, ciclofosfamida ${ }^{20}$ y plasmaféresis ${ }^{24}$. En caso de reactivación viral (CMV, HHV-6), el uso de ganciclovir es una alternativa ${ }^{14}$. Basado en los altos niveles de interleuquina-5 (citoquina Th2 y factor de diferenciación eosinófilo-específico) observados en DRESS, mepolizumab, un anticuerpo monoclonal anti-IL-5, se encuentra en investigación ${ }^{25}$. Otro reciente reporte de secuenciación de ARN de célula individual (scRNA-seq) en piel y sangre de un paciente con DRESS refractario a corticoides logró la identificación de la vía de señalización JAK-STAT como potencial blanco terapéutico: tofacitinib, un inhibidor JAK-kinasa, logró suprimir la proliferación de células T inducida por la droga culpable ${ }^{26}$. En nuestra paciente, la rápida suspensión de sulfasalazina mejoró el cuadro clínico, no siendo necesario corticoides ni otras terapias alternativas.

\section{Conclusión}

El síndrome de DRESS es un cuadro asociado al uso de algunos fármacos, con un espectro clínico variado y potencialmente letal. El conocimiento de esta entidad y la sospecha clínica es fundamental para mejorar el manejo y pronóstico de los pacientes, siendo necesario siempre investigar su historial farmacológico. En el caso de sulfasalazina, es un evento adverso que debe considerarse al inicio del tratamiento y ante la aparición de fiebre y exanetma acompañado o no de otros síntomas en las primeras semanas de su uso.

\section{Referencias}

1. Bocquet H, Boagot M, Roujeau JC. Drug-induced pseudolymphoma and drug hypersensitivity (Drug rash with eosinophilia and systemic symptoms: DRESS). Semin Cutan Med Surg 1996; 15 (4): 250-7.

2. Cacoub P, Musette P, Descamps V, Meyer O, Speirs C, Finzi L, et al. The DRESS syndrome: a literature review. Am J Med 2011; 124 (7): 588-97.

3. Peyrie`re H, Dereure O, Breton H, Demoly P, Cociglio M, Blayac JP, et al. Variability in the clinical pattern of cutaneous side-effects of drugs with systemic symptoms: does a DRESS syndrome really exist? Br J Dermatol 2006; 155 (2): 422-8.

4. Duong TA, Valeyrie-Allanore L, Wolkenstein P, Chosidow O. Severe cutaneous adverse reactions to drugs. Lancet 2017; 390 (10106): 1996-2011.

5. Gennis MA, Vemuri R, Burns EA, Hill JV, Miller MA, Spielberg SP. Familial occurrence of hypersensitivity to phenytoin. Am J Med 1991; 91 (6): 631-4.

6. Fiszenson-Albala F, Auzerie V, Mahe E, Farinotti R, Durand-Stococco C, Crickx B, et al. A 6-month prospective survey of cutaneous drug reactions in a hospital setting. Br J Dermatol 2003; 149 (5): 1018-22.

7. Wolfson AR, Zhou L, Li Y, Phadke NA, Chow OA, Blumenthal KG. Drug Reaction with Eosinophilia and Systemic Symptoms (DRESS) Syndrome Identified in the Electronic Health Record Allergy Module. J Allergy Clin Immunol Pract 2019; 7 (2): 633-40.

8. Kardaun SH, Sekula P, Veleyrie-Allanore L, Liss Y, Chu CY, Creamer D, et al. Drug reaction with eosinophilia and systemic symptoms (DRESS): an original multisystem adverse drug reaction. Results from the prospective RegiSCAR study. Br J Dermatol 2013; 169 (5): 1071-80.

9. Shiohara T, Iijima M, Ikezawa Z, Hashimoto K. The diagnosis of a DRESS syndrome has been sufficiently established on the basis of typical clinical features and viral reactivations. Br J Dermatol 2007; 156 (5): 1083-4.

10. Shiohara T, Mizukawa Y. Drug-induced hypersensitivity syndrome (DiHS)/drug reaction with eosinophilia and 
systemic symptoms (DRESS): An update in 2019. Allergol Int 2019; 68 (3): 301-8.

11. Adwan MH. Drug Reaction with Eosinophilia and Systemic Symptoms (DRESS) Syndrome and the Rheumatologist. Curr Rheumatol Rep 2017; 19 (1): 3.

12. Bellón T. Mechanisms of Severe Cutaneous Adverse Reactions: Recent Advances. Drug Saf 2019; 42 (8): 973-92.

13. Kano Y, Shiohara T. The variable clinical picture of drug-induced hypersensitivity syndrome/drug rash whit eosinophilia and systemic symptoms in relation to the eliciting drug. Immunol and Allergy Clin North Am 2009; 29 (3): 481-501.

14. Michel F, Navellou JC, Ferraud D, Toussirot E, Wendling D. DRESS syndrome in a patient on sulfasalazine for rheumatoid arthritis. Join Bone Spine 2005; 72 (1): 82-5.

15. Shear NH, Spielberg SP, Grant DM, Tank BK, Kalow W. Differences in metabolism of sulfonamides predisposing to idiosyncratic toxicity. Ann Intern Med 1986; 105 (2): 179-84.

16. Naranjo CA, Busto U, Sellers EM, Sandor P, Ruiz I, Roberts EA, et al. A method for estimating the probability of adverse drug reactions. Clin Pharmacol Ther 1981; 30 (2): 239-45.

17. Mizukawa Y, Hirahara K, Kano Y, Shiohara T. Drug-induced hypersensitivity syndrome/drug reaction with eosinophilia and systemic symptoms severity score: A useful tool for assessing disease severity and predicting fatal cytomegalovirus disease. J Am Acad Dermatol 2019; 80 (3): 670-8.

18. Phatharacharukul P, Klaewsongkram J. A case of sulfasalazine-induced hypersensitivity syndrome confirmed by enzyme-linked immunospot assay. Allergy Asthma Immunol Res 2013; 5 (6): 415-7.
19. Kato K, Kawase A, Azukizawa H, Hanafusa T, Nakagawa $\mathrm{Y}$, Murota $\mathrm{H}$, et al. Novel interferon- $\gamma$ enzyme-linked immunoSpot assay using activated cells for identifying hypersensitivity-inducing drug culprits. J Dermatol Sci 2017; 86 (3): 222-9.

20. Descamps V, Ben-Saïd B, Sassolas B, Truchetet F, Avenel-Audran M, Girardin P, et al. Management of drug reaction with eosinophilia and systemic symptoms (DRESS). Ann Dermatol Venereol 2010; 137 (11): 7038.

21. Moling O, Tappeiner L, Piccin A, Pagani E, Rossi P, Rimenti G, et al. Treatment of DIHS/DRESS syndrome with combined $\mathrm{N}$-acetylcysteine, prednisone, and valganciclovir - a hypothesis. Med Sci Monit 2012; 18 (7): 57-62.

22. Singer EM, Wanat KA, Rosenbach MA. A case of recalcitrant DRESS syndrome with multiple autoimmune sequelae treated with intravenous immunoglobulins. JAMA Dermatol 2013; 149 (4): 494-5.

23. Kirchhof MG, Wong A, Dutz JP. Cyclosporine treatment of drug-induced hypersensitivity syndrome. JAMA Dermatol. 2016; 152: 1254-7.

24. Alexander T, Iglesia E, Park Y, Duncan D, Peden D, Sheikh S, et al. Severe DRESS syndrome managed with therapeutic plasma exchange. Pediatrics 2013; 131 (3): 945-9.

25. Ange N, Alley S, Fernando SL, Coyle L, Yun J. Drug Reaction with Eosinophilia and Systemic Symptoms (DRESS) síndrome successfully treated with mepolizumab. J Allergy Clin Immunol Pract 2018; 6 (3): 1059-60.

26. Kim D, Kobayashi T, Voisin B, Jo JH, Sakamoto K, Jin $\mathrm{SP}$, et al. Targeted therapy guided by single-cell transcriptomic analysis in drug-induced hypersensitivity syndrome: a case report. Nat Med 2020; 26 (2): 236-43. 